\title{
Exact Solutions of Brinkman Type Fluid Between Side Walls Over an Infinite Plate
}

\author{
Muhammad Asif ${ }^{a}$, Sami Ul Haq ${ }^{b, *}$, Saeed $\operatorname{Islam}^{a}$ \\ Department of Mathematics \\ ${ }^{a}$ Abdul Wali Khan University, Mardan, Khyber Pakhtunkhwa, Pakistan. \\ ${ }^{b}$ Islamia college University, Peshawar, Khyber Pakhtunkhwa, Pakistan. \\ * Corresponding Author Email: samiulhaq@icp.edu.pk
}

\begin{abstract}
In this paper Brinkman type fluid over an infinite plate between side walls is being investigated. The flow is generated by oscillating shear stress of the bottom plate and the solutions are obtained by using Fourier integral transformation. The obtained results are presented in steady and transient states for both sin and cos shear stresses. The general solutions are reduced to some special cases corresponding, namely to the Brinkman type fluid over an infinite plate and flow of a Newtonian viscous fluid. Graphical illustrations are carried out to have in depth analysis of the involved physical parameters.
\end{abstract}

\section{Keywords}

Brinkman type fluid, Fourier Integral transformation, side walls, oscillating shear stress.

\section{Introduction}

In the literature fluids are mainly divided into two types, namely Newtonian and non-Newtonian. Here the non-Newtonian fluids are of very sophisticated structure, available in different models in literature. Brinkman type fluid is one of these complex models suggested by H. C. Brinkman [1,2]. He showed that the flow through a highly permeable media is Brinkman type fluid. An incompressible fluid of viscous nature flowing over a highly porous media is known as Brinkman type fluid [3]. Vijay Kumar Varma and Syam BaBu have discussed the steady flow of incompressible fluid in a channel of different permeabilities media applying uniform magnetic field [4]. C. T. Hsu and P. Cheng [5] have 
considered Brinkman model for the analysis of effect of boundaries a convection of porosity to the semi infinite plate in vertical position with the variation of power law. The Darcy's equation [6] has been modified into a special case named Brinkman equation, who developed the modified form, because Darcy's equation has not related viscous stress tensor. Rajagopal [7] has derived more strategist governing equation by taking flow through porous media. Ali et al. [8] have provided a solution to the flow of Brinkman type fluid induced by the oscillating plate by using Laplace transformation. Brinkman type fluid has been studied in fractional form using Caputo fractional derivative definition. The model has been solved using Laplace transformation with and without heat and mass transfer. Brinkman model with MHD over a plate in vertical position enclosed by porous medium has been solved using method of Laplace transformation taking different types of nano particles into experiment [3]. Brinkman type equation has been solved using multi-scale hybrid mixed(MHM) method [9]. Free convectional flow in vertical channel has many applications in engineering and industrial processes like to extend the working time of lubricants, functioning time of parts of machinery and electronic materials etc. The Luminar totally developed flow with mixed convection has been analyzed by Tao [10]. Hartmann [11] has analyzed MHD flow of an incompressible, viscous fluid between two plates which are parallel to each other and transverse magnetic field has been applied. Flow of Maxwell fluid [12] between two side walls perpendicular to the plate where the flow is due to the acceleration in the plate has been analyzed by the help of Fourier transformation. Maxwell fluid flowing unsteadily over a flat plate among two side walls orthogonal to the plate and parallel to each other studied by Akhtar et al. [13], while the exact solution has been derived by using Fourier transformation. Vieru et al. [14] have studied and found the exact solutions for second grade fluid over an infinite plate among two side walls as of the same geometry while accelerated shear stress has been applied to the fluid by the help of Fourier transformation. In 2010 yao and Liu [15] have used Fourier transformation for finding the effect of side walls of second grade fluid flowing unsteadily over a plane wall. Fetecau et al. [16] have taken help from the method of Fourier transformation for finding the effect of side walls on the movement (motion) of the fluid which viscous in nature, while flow is produced by the oscillating shear stress. So far different authors like [17-21] have used Fourier transformation to obtain the effects of 
side walls in different forms to the fluids in unlike conditions.

Keeping in view all the literature discussed above we observe that the channel flow Brinkman type fluid subjected to oscillating shear stress " $\rho \mathrm{e}^{i w t "}$ have not been discussed. Solutions of the problem are obtained in sin oscillation, cos oscillation, transient and steady state. Different physical parameters have been discussed graphically.

\section{Problem Formulation}

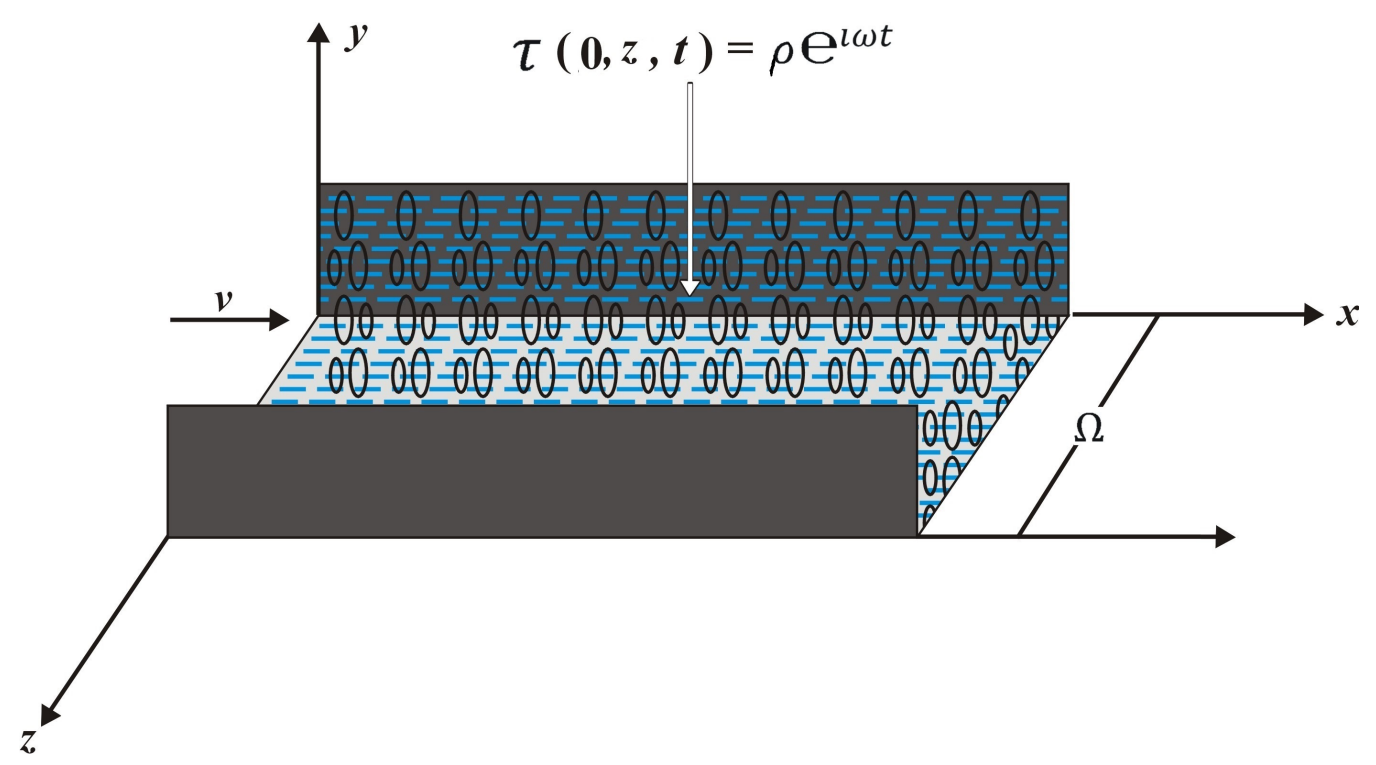

Figure 1: geometry of the problem

Consider a non-Newtonian incompressible Brinkman type fluid present up at an infinite plate enclosed by two side walls parallel to each other and perpendicular to the horizontal plate. For the time $t=0^{+}$, the fluid is being induced by the oscillating shear stress $\rho \mathrm{e}^{i w t}$ by the plate due to which produce velocity in the form

$$
\boldsymbol{v}=v(y, z, t) \mathbf{i}
$$

where $\mathbf{i}$ stands for unit vector.

The governing equation is

$$
\rho\left[\frac{\partial v(y, z, t)}{\partial t}+\beta v(y, z, t)\right]=\mu\left[\frac{\partial^{2} v(y, z, t)}{\partial y^{2}}+\frac{\partial^{2} v(y, z, t)}{\partial z^{2}}\right],
$$

here $\beta$ shows the Brinkman parameter while the adequate conditions (initial and boundary) are

$$
v(y, z, 0)=0, \quad \text { for } y>0 \text { and } z \in[0, \Omega]
$$




$$
\begin{gathered}
v(y, 0, t)=v(y, \Omega, t)=0 ; \quad \text { for } y, t>0, \\
\tau(y, z, t)=\left.\frac{\partial v(y, z, t)}{\partial y}\right|_{y=0}=f \mathrm{e}^{i w t}
\end{gathered}
$$

above relation(3) shows the shear stress which is non-trivial, $w$ stands for frequency of the plate, $f$ shows constant parameter

$$
v(y, z, t) \text { and } \frac{\partial v(y, z, t)}{\partial y} \rightarrow 0 \quad \text { as } y \rightarrow \infty, \quad z \in[0, \Omega] \text { fort }>0
$$

\section{Problem Solution}

Multiplying $\sqrt{\frac{2}{\pi}} \cos (\varsigma y) \sin \left(\gamma_{r} z\right)$ and the result is being integrated as y and z respectively keeping in view the given conditions we acquire the below differential equation

$$
\left(\frac{\partial}{\partial t}+\beta\right) v_{c r}(\varsigma, t)+\nu\left[\varsigma^{2}+\gamma_{r}^{2}\right] v_{c r}(\varsigma, t)=\nu \sqrt{\frac{2}{\pi}} \frac{\rho}{\phi} \mathrm{e}^{i w t}\left(\frac{(-1)^{r}-1}{\gamma_{r}}\right)
$$

as

$$
v_{c r}(\varsigma, t)=\sqrt{\frac{2}{\pi}} \int_{0}^{\infty} \int_{0}^{\Omega} v(y, z, t) \cos (y \varsigma) \sin \left(\gamma_{r} z\right) d z d y \quad \text { for } \quad r=1,2,3, \ldots
$$

the initial condition is satisfied by $v(y, z, t)$ as

$$
v_{c r}(\varsigma, 0)=0 \text { for } \varsigma>0 \text { and } r=1,2,3, \ldots
$$

as eq. (5) is an ordinary differential equation in $t$, solving it using integration factor and initial condition given by eq. (6) we obtain the result as

$$
\begin{aligned}
v_{c r}(\varsigma, t)=\sqrt{\frac{2}{\pi}} \frac{\nu \rho}{\phi} \frac{\left((-1)^{r}-1\right)}{\gamma_{r}}\left[\frac{\left(\frac{\beta}{\nu}\left[\varsigma^{2}+\gamma_{r}^{2}\right]\right) \cos (w t)+\frac{w}{\nu} \sin (w t)}{\left(\frac{\beta}{\nu}+\left[\varsigma^{2}+\gamma_{r}^{2}\right]\right)^{2}+\left(\frac{w}{\nu}\right)^{2}}\right. \\
+i \frac{\left(\frac{\beta}{\nu}\left[\varsigma^{2}+\gamma_{r}^{2}\right]\right) \sin (w t)-\frac{w}{\nu} \cos (w t)}{\left(\frac{\beta}{\nu}+\left[\varsigma^{2}+\gamma_{r}^{2}\right]\right)^{2}+\left(\frac{w}{\nu}\right)^{2}} \\
+\frac{\left(i \frac{w}{\nu}-\left(\frac{\beta}{\nu}+\left[\varsigma^{2}+\gamma_{r}^{2}\right]\right)\right.}{\left(\frac{\beta}{\nu}+\left[\varsigma^{2}+\gamma_{r}^{2}\right]\right)^{2}+\left(\frac{w}{\nu}\right)^{2}} \mathrm{e}^{\left.-\left(\frac{\beta}{\nu}+\left[\varsigma^{2}+\gamma_{r}^{2}\right]\right) t\right] .}
\end{aligned}
$$


applying Fourier inverse transformation we obtain

$$
\begin{aligned}
& v(y, z, t)=\frac{8 \rho}{\pi \Omega \phi} \sum_{r=0}^{\infty} \frac{\sin \left(\gamma_{s} z\right)}{\gamma_{s}} \int_{0}^{\infty}\left[\frac{\left(\frac{\beta}{\nu}\left[\varsigma^{2}+\gamma_{s}^{2}\right]\right) \cos (w t)+\frac{w}{\nu} \sin (w t)}{\left(\frac{\beta}{\nu}+\left[\varsigma^{2}+\gamma_{s}^{2}\right]\right)^{2}+\left(\frac{w}{\nu}\right)^{2}}\right. \\
& +i \frac{\left(\frac{\beta}{\nu}\left[\varsigma^{2}+\gamma_{s}^{2}\right]\right) \sin (w t)-\frac{w}{\nu} \cos (w t)}{\left(\frac{\beta}{\nu}+\left[\varsigma^{2}+\gamma_{s}^{2}\right]\right)^{2}+\left(\frac{w}{\nu}\right)^{2}} \\
& \left.\left.+\frac{\left(i \frac{w}{\nu}-\left(\frac{\beta}{\nu}+\left[\varsigma^{2}+\gamma_{r}^{2}\right]\right)\right.}{\left(\frac{\beta}{\nu}+\left[\varsigma^{2}+\gamma_{r}^{2}\right]\right)^{2}+\left(\frac{w}{\nu}\right)^{2}}\right) \mathrm{e}^{-\left(\frac{\beta}{\nu}+\left[\varsigma^{2}+\gamma_{r}^{2}\right]\right) t}\right] \cos (y \varsigma) d \varsigma,
\end{aligned}
$$

here $s=2 r-1$, taking $\Omega=2 h$ and switching the origin by taking $z=z^{\prime}+h$ and expelling the $\alpha$ notation, the above equation gives

$$
\begin{aligned}
v(y, z, t)=\frac{4 \rho}{\pi h \phi} \sum_{s=1}^{\infty}( & -1)^{s} \frac{\cos \left(\lambda_{s} z\right)}{\lambda_{s}} \int_{0}^{\infty}\left[\frac{\left(\frac{\beta}{\nu}\left[\varsigma^{2}+\lambda_{s}^{2}\right]\right) \cos (w t)+\frac{w}{\nu} \sin (w t)}{\left(\frac{\beta}{\nu}+\left[\varsigma^{2}+\lambda_{s}^{2}\right]\right)^{2}+\left(\frac{w}{\nu}\right)^{2}}\right. \\
+ & i \frac{\left(\frac{\beta}{\nu}\left[\varsigma^{2}+\lambda_{s}^{2}\right]\right) \sin (w t)-\frac{w}{\nu} \cos (w t)}{\left(\frac{\beta}{\nu}+\left[\varsigma^{2}+\lambda_{s}^{2}\right]\right)^{2}+\left(\frac{w}{\nu}\right)^{2}} \\
& +\frac{\left(i \frac{w}{\nu}-\left(\frac{\beta}{\nu}+\left[\varsigma^{2}+\gamma_{r}^{2}\right]\right)\right.}{\left(\frac{\beta}{\nu}+\left[\varsigma^{2}+\gamma_{r}^{2}\right]\right)^{2}+\left(\frac{w}{\nu}\right)^{2}} \mathrm{e}^{\left.-\left(\frac{\beta}{\nu}+\left[\varsigma^{2}+\gamma_{r}{ }^{2}\right]\right) t\right]} \cos (y \varsigma) d \varsigma .
\end{aligned}
$$

using these identities

$$
\begin{gathered}
\frac{w}{\nu} \int_{s=0}^{\infty} \frac{\cos (y \varsigma)}{\left(\varsigma^{2}+\lambda_{s}^{2}\right)^{2}+\left(\frac{w}{\nu}\right)^{2}} d \varsigma=\frac{\pi}{2} \frac{C_{s} \cos \left(y C_{s}\right)+D_{s} \sin \left(y C_{s}\right)}{C_{s}^{2}+D_{s}^{2}}, \\
\int_{0}^{\infty} \frac{\left(\varsigma^{2}+\lambda_{s}^{2}\right) \cos (y \varsigma)}{\left(\varsigma^{2}+\lambda_{s}^{2}\right)^{2}+\left(\frac{w}{\nu}\right)^{2}} d \varsigma=\frac{\pi}{2} \frac{D_{s} \cos \left(y C_{s}\right)-C_{s} \sin \left(y C_{s}\right)}{C_{s}^{2}+D_{s}^{2}} \mathrm{e}^{-y D_{s}},
\end{gathered}
$$

with

$2 C_{s}^{2}=\sqrt{\lambda_{s}^{4}+\left(\frac{w}{\nu}\right)^{2}}-\lambda_{s}^{2}$ and $2 D_{s}^{2}=\sqrt{\lambda_{s}^{4}+\left(\frac{w}{\nu}\right)^{2}}+\lambda_{s}^{2}$ from [16]

the simplified form of velocity $v(y, z, t)$ can be obtained as

$$
\begin{aligned}
v(y, z, t)=\frac{2 \rho}{h \phi} \sum_{s=0}^{\infty} & (-1) \frac{\operatorname{sos}\left(\lambda_{s} z\right)}{\lambda_{s}} \frac{\mathrm{e}^{\left(-y D_{s}\right)}}{C_{s}^{2}+D_{s}^{2}} \\
& {\left[D_{s} \cos \left(w t-y C_{s}\right)+C_{s} \sin \left(w t-y C_{s}\right)\right.} \\
+ & \left.i\left(D_{s} \sin \left(w t-y C_{s}\right)-C_{s} \cos \left(w t-y C_{s}\right)\right)\right] \\
& +\frac{4 \rho}{\pi h \phi} \sum_{s=0}^{\infty}(-1)^{s} \frac{\cos \left(\lambda_{s} z\right)}{\lambda_{s}} \\
& \left.\int_{0}^{\infty} \frac{\left(i \frac{w}{\nu}-\left(\frac{\beta}{\nu}+\left[\varsigma^{2}+\lambda_{s}^{2}\right]\right)\right.}{\left(\frac{\beta}{\nu}+\left[\varsigma^{2}+\lambda_{s}^{2}\right]\right)^{2}+\left(\frac{w}{\nu}\right)^{2}}\right) \mathrm{e}^{-\left(\frac{\beta}{\nu}+\left[\varsigma^{2}+\lambda_{s}{ }^{2}\right]\right) t} \cos (y \varsigma) d \varsigma,
\end{aligned}
$$


eq(12) can be reduced in polar form as

$$
\begin{array}{r}
v(y, z, t)=\frac{2 \rho}{h \phi} \sum_{s=0}^{\infty}(-1)^{s} \frac{\cos \left(\lambda_{s} z\right)}{\lambda_{s}} \frac{\mathrm{e}^{\left[-y D_{s}\right]}}{C_{s}^{2}+D_{s}^{2}}\left[\cos \left(\psi-\Phi_{s}\right)+i \sin \left(\psi-\Phi_{s}\right)\right] \\
+\frac{4 \rho}{\pi h \phi} \sum_{s=0}^{\infty}(-1)^{s} \frac{\cos \left(\lambda_{s} z\right)}{\lambda_{s}} \\
\int_{0}^{\infty} \frac{\left(i \frac{w}{\nu}-\left(\frac{\beta}{\nu}+\left[\varsigma^{2}+\lambda_{s}^{2}\right]\right)\right.}{\left(\frac{\beta}{\nu}+\left[\varsigma^{2}+\lambda_{s}^{2}\right]\right)^{2}+\left(\frac{w}{\nu}\right)^{2}} \mathrm{e}^{-\left(\frac{\beta}{\nu}+\left[\varsigma^{2}+\lambda_{s}{ }^{2}\right]\right) t} \cos (y \varsigma) d \varsigma,
\end{array}
$$

where $\psi=w t-y C_{s}$.

Solution given in eq. (13) is verified by using given(initial and boundary) conditions, eq. (13) can be written in terms of steady and transient parts as

$$
\begin{aligned}
v_{S}(y, z, t)=\frac{2 \rho}{h \phi} \sum_{s=0}^{\infty}(-1)^{s} \frac{\cos \left(\lambda_{s} z\right)}{\lambda_{s}} \frac{\mathrm{e}^{\left[-y D_{s}\right]}}{C_{s}^{2}+D_{s}^{2}} & \\
& \begin{array}{r}
{\left[D_{s} \cos \left(w t-y C_{s}\right)+C_{s} \sin \left(w t-y C_{s}\right)\right.} \\
\left.\quad+i\left(D_{s} \sin \left(w t-y C_{s}\right)-C_{s} \cos \left(w t-y C_{s}\right)\right)\right],
\end{array}
\end{aligned}
$$

and

$$
\begin{aligned}
v_{T}(y, z, t)=\frac{4 \rho}{\pi h \phi} \sum_{s=0}^{\infty}(-1)^{s} \frac{\cos \left(\lambda_{s} z\right)}{\lambda_{s}} & \\
& \left.\int_{0}^{\infty} \frac{\left(i \frac{w}{\nu}-\left(\frac{\beta}{\nu}+\left[\varsigma^{2}+\lambda_{s}{ }^{2}\right]\right)\right.}{\left(\frac{\beta}{\nu}+\left[\varsigma^{2}+\lambda_{s}^{2}\right]\right)^{2}+\left(\frac{w}{\nu}\right)^{2}}\right) \mathrm{e}^{-\left(\frac{\beta}{\nu}+\left[\varsigma^{2}+\lambda_{s}{ }^{2}\right]\right) t} \cos (y \varsigma) d \varsigma,
\end{aligned}
$$

while eq. (13) can be separated into real and imaginary parts as

$$
\begin{aligned}
& v_{c}(y, z, t)=\frac{2 \rho}{h \phi} \sum_{s=0}^{\infty}(-1)^{s} \frac{\cos \left(\lambda_{s} z\right)}{\lambda_{s}} \frac{\mathrm{e}^{\left[-y D_{s}\right]}\left(D_{s} \cos \left(w t-y C_{s}\right)+C_{s} \sin \left(w t-y C_{s}\right)\right)}{C_{s}^{2}+D_{s}^{2}} \\
&-\frac{4 \rho}{\pi h \phi} \sum_{s=0}^{\infty}(-1)^{s} \frac{\cos \left(\lambda_{s} z\right)}{\lambda_{s}} \\
&\left.\int_{0}^{\infty} \frac{\left(\left(\frac{\beta}{\nu}+\left[\varsigma^{2}+\lambda_{s}{ }^{2}\right]\right)\right.}{\left(\frac{\beta}{\nu}+\left[\varsigma^{2}+\lambda_{s}{ }^{2}\right]\right)^{2}+\left(\frac{w}{\nu}\right)^{2}}\right) \mathrm{e}^{-\left(\frac{\beta}{\nu}+\left[\varsigma^{2}+\lambda_{s}{ }^{2}\right]\right) t} \cos (y \varsigma) d \varsigma, \quad(16)
\end{aligned}
$$




$$
\begin{aligned}
& v_{s}(y, z, t)=\frac{2 \rho}{h \phi} \sum_{s=0}^{\infty}(-1)^{s} \frac{\cos \left(\lambda_{s} z\right)}{\lambda_{s}} \frac{\mathrm{e}^{\left[-y D_{s}\right]}\left(D_{s} \sin \left(w t-y C_{s}\right)-C_{s} \cos \left(w t-y C_{s}\right)\right)}{C_{s}^{2}+D_{s}^{2}} \\
&+\frac{4 \rho}{\pi h \phi} \sum_{s=0}^{\infty}(-1)^{s} \frac{\cos \left(\lambda_{s} z\right)}{\lambda_{s}} \\
&\left.\frac{w}{\nu} \int_{0}^{\infty} \frac{\cos (y \varsigma)}{\left(\frac{\beta}{\nu}+\left[\varsigma^{2}+\lambda_{s}^{2}\right]\right)^{2}+\left(\frac{w}{\nu}\right)^{2}}\right) \mathrm{e}^{-\left(\frac{\beta}{\nu}+\left[\varsigma^{2}+\lambda_{s}^{2}\right]\right) t} d \varsigma . \quad \text { (17) }
\end{aligned}
$$

here eq. (16) shows cos oscillation while eq. (17) shows sin oscillation of the velocity distribution.

The shear stress exerted by the bottom plate to the fluid namely $\tau(y, z, t)$ is

$$
\begin{gathered}
\tau(y, z, t)=\frac{2 \rho}{h} \sum_{s=0}^{\infty}(-1)^{s} \frac{\cos \left(\lambda_{s} z\right)}{\lambda_{s}} \mathrm{e}^{\left[-y D_{s}\right]}\left[\cos \left(w t-y C_{s}\right)+i \sin \left(w t-y C_{s}\right)\right] \\
-\frac{4 \rho}{\pi h} \sum_{s=0}^{\infty}(-1)^{s} \frac{\cos \left(\lambda_{s} z\right)}{\lambda_{s}} \\
\int_{0}^{\infty} \frac{\left(i \frac{w}{\nu}-\left(\frac{\beta}{\nu}+\left[\varsigma^{2}+\lambda_{s}^{2}\right]\right)\right.}{\left(\frac{\beta}{\nu}+\left[\varsigma^{2}+\lambda_{s}^{2}\right]\right)^{2}+\left(\frac{w}{\nu}\right)^{2}} \mathrm{e}^{-\left(\frac{\beta}{\nu}+\left[\varsigma^{2}+\lambda_{s}^{2}\right]\right) t} \varsigma \sin (y \varsigma) d \varsigma .
\end{gathered}
$$

eq. (18) can be written separately in sin and cos oscillations form as

$$
\begin{aligned}
& \tau_{c}(y, z, t)=\frac{2 \rho}{h} \sum_{s=0}^{\infty}(-1)^{s} \frac{\cos \left(\lambda_{s} z\right)}{\lambda_{s}} \mathrm{e}^{\left[-y D_{s}\right]}\left[\cos \left(w t-y C_{s}\right)\right] \\
& +\frac{4 \rho}{\pi h} \sum_{s=0}^{\infty}(-1)^{s} \frac{\cos \left(\lambda_{s} z\right)}{\lambda_{s}} \\
& \left.\int_{0}^{\infty} \frac{\left(\frac{\beta}{\nu}+\left[\varsigma^{2}+\lambda_{s}^{2}\right]\right)}{\left(\frac{\beta}{\nu}+\left[\varsigma^{2}+\lambda_{s}^{2}\right]\right)^{2}+\left(\frac{w}{\nu}\right)^{2}}\right) \mathrm{e}^{-\left(\frac{\beta}{\nu}+\left[\varsigma^{2}+\lambda_{s}{ }^{2}\right]\right) t} \varsigma \sin (y \varsigma) d \varsigma, \\
& \tau_{s}(y, z, t)=\frac{2 \rho}{h} \sum_{s=0}^{\infty}(-1)^{s} \frac{\cos \left(\lambda_{s} z\right)}{\lambda_{s}} \mathrm{e}^{\left[-y D_{s}\right]}\left[\sin \left(w t-y C_{s}\right)\right] \\
& -\frac{4 \rho}{\pi h} \sum_{s=0}^{\infty}(-1)^{s} \frac{\cos \left(\lambda_{s} z\right)}{\lambda_{s}} \\
& \int_{0}^{\infty} \frac{\frac{w}{\nu}}{\left(\frac{\beta}{\nu}+\left[\varsigma^{2}+\lambda_{s}^{2}\right]\right)^{2}+\left(\frac{w}{\nu}\right)^{2}} \mathrm{e}^{-\left(\frac{\beta}{\nu}+\left[\varsigma^{2}+\lambda_{s}^{2}\right]\right) t} \varsigma \sin (y \varsigma) d \varsigma .
\end{aligned}
$$




\section{Special Cases}

\subsection{When $h \rightarrow \infty$ ( Maximizing distance between side walls)}

If we increase the distance between the side walls means $h \rightarrow \infty$, we obtain the solutions of the eqs. (16),(17),(19) and (20) as

$$
\begin{aligned}
& v_{c}(y, t)=\frac{-\rho \sqrt{2 \nu}}{\phi} \frac{D_{s} \cos \left(w t-y C_{s}\right)+C_{s} \sin \left(w t-y C_{s}\right)}{C_{s}^{2}+D_{s}^{2}} \mathrm{e}^{-y D_{s}} \\
& +\frac{2 \rho}{\phi \pi} \int_{0}^{\infty} \frac{\left(\left(\frac{\beta}{\nu}+\left[\varsigma^{2}+\lambda_{s}^{2}\right]\right)\right.}{\left(\frac{\beta}{\nu}+\left[\varsigma^{2}+\lambda_{s}^{2}\right]\right)^{2}+\left(\frac{w}{\nu}\right)^{2}} \mathrm{e}^{-\left(\frac{\beta}{\nu}+\left[\varsigma^{2}+\lambda_{s}^{2}\right]\right) t} \cos (y \varsigma) d \varsigma, \\
& v_{s}(y, t)=\frac{-\rho \sqrt{2 \nu}}{\phi} \frac{D_{s} \sin \left(w t-y C_{s}\right)-C_{s} \cos \left(w t-y C_{s}\right)}{C_{s}^{2}+D_{s}^{2}} \mathrm{e}^{-y D_{s}} \\
& -\frac{2 \rho}{\phi \pi} \frac{w}{\nu} \int_{0}^{\infty} \frac{\cos (y \varsigma)}{\left(\frac{\beta}{\nu}+\left[\varsigma^{2}+\lambda_{s}^{2}\right]\right)^{2}+\left(\frac{w}{\nu}\right)^{2}} \mathrm{e}^{-\left(\frac{\beta}{\nu}+\left[\varsigma^{2}+\lambda_{s}{ }^{2}\right]\right) t} d \varsigma, \\
& \tau_{c}(y, t)=\frac{-\rho \sqrt{2 \nu}}{\phi} \frac{D_{s} \cos \left(w t-y C_{s}\right)+C_{s} \sin \left(w t-y C_{s}\right)}{C_{s}^{2}+D_{s}^{2}} \mathrm{e}^{-y D_{s}} \\
& +\frac{2 \rho}{\phi \pi} \int_{0}^{\infty} \frac{\left(\left(\frac{\beta}{\nu}+\left[\varsigma^{2}+\lambda_{s}^{2}\right]\right)\right.}{\left(\frac{\beta}{\nu}+\left[\varsigma^{2}+\lambda_{s}^{2}\right]\right)^{2}+\left(\frac{w}{\nu}\right)^{2}} \mathrm{e}^{-\left(\frac{\beta}{\nu}+\left[\varsigma^{2}+\lambda_{s}^{2}\right]\right) t} \cos (y \varsigma) d \varsigma, \\
& \tau_{s}(y, t)=\frac{-\rho \sqrt{2 \nu}}{\phi} \frac{D_{s} \sin \left(w t-y C_{s}\right)-C_{s} \cos \left(w t-y C_{s}\right)}{C_{s}^{2}+D_{s}^{2}} \mathrm{e}^{-y D_{s}} \\
& -\frac{2 \rho}{\phi \pi} \frac{w}{\nu} \int_{0}^{\infty} \frac{\cos (y \varsigma)}{\left(\frac{\beta}{\nu}+\left[\varsigma^{2}+\lambda_{s}^{2}\right]\right)^{2}+\left(\frac{w}{\nu}\right)^{2}} \mathrm{e}^{-\left(\frac{\beta}{\nu}+\left[\varsigma^{2}+\lambda_{s}^{2}\right]\right) t} d \varsigma .
\end{aligned}
$$

\section{2 when $\beta \rightarrow 0$ (Ignoring the Brinkman parameter)}

Consider that the Brinkman parameter $\beta$ approaches to zero the eqs. (16), (17), (19) and (20) will give us the solutions as derived by fetecau et al. [16]

$$
\begin{aligned}
v_{c}(y, z, t)=\frac{2 \rho}{h \phi} \sum_{s=0}^{\infty}(-1)^{s} \frac{\cos \left(\lambda_{s} z\right)}{\lambda_{s}} \frac{\mathrm{e}^{\left[-y D_{s}\right]}\left(D_{s} \cos \left(w t-y C_{s}\right)+C_{s} \sin \left(w t-y C_{s}\right)\right)}{C_{s}^{2}+D_{s}^{2}} \\
-\frac{4 \rho}{\pi h \phi} \sum_{s=0}^{\infty}(-1)^{s} \frac{\cos \left(\lambda_{s} z\right)}{\lambda_{s}} \\
\int_{0}^{\infty} \frac{\left[\varsigma^{2}+\lambda_{s}{ }^{2}\right]}{\left(\varsigma^{2}+\lambda_{s}{ }^{2}\right)^{2}+\left(\frac{w}{\nu}\right)^{2}} \mathrm{e}^{-\left(\varsigma^{2}+\lambda_{s}{ }^{2}\right) t} \cos (y \varsigma) d \varsigma, \quad(25)
\end{aligned}
$$




$$
\begin{aligned}
& v_{s}(y, z, t)=\frac{2 \rho}{h \phi} \sum_{s=0}^{\infty}(-1)^{s} \frac{\cos \left(\lambda_{s} z\right)}{\lambda_{s}} \frac{\mathrm{e}^{\left[-y D_{s}\right]}\left(D_{s} \sin \left(w t-y C_{s}\right)-C_{s} \cos \left(w t-y C_{s}\right)\right)}{C_{s}^{2}+D_{s}^{2}} \\
& +\frac{4 \rho}{\pi h \phi} \sum_{s=0}^{\infty}(-1)^{s} \frac{\cos \left(\lambda_{s} z\right)}{\lambda_{s}} \\
& \frac{w}{\nu} \int_{0}^{\infty} \frac{\cos (y \varsigma)}{\left(\varsigma^{2}+\lambda_{s}^{2}\right)^{2}+\left(\frac{w}{\nu}\right)^{2}} \mathrm{e}^{-\left(\varsigma^{2}+\lambda_{s}^{2}\right) t} d \varsigma, \\
& \tau_{c}(y, z, t)=\frac{2 \rho}{h} \sum_{s=0}^{\infty}(-1)^{s} \frac{\cos \left(\lambda_{s} z\right)}{\lambda_{s}} \mathrm{e}^{-y D_{s}}\left[\cos \left(w t-y C_{s}\right)\right] \\
& +\frac{4 \rho}{\pi h} \sum_{s=0}^{\infty}(-1)^{s} \frac{\cos \left(\lambda_{s} z\right)}{\lambda_{s}} \\
& \int_{0}^{\infty} \frac{\left[\varsigma^{2}+\lambda_{s}^{2}\right]}{\left(\varsigma^{2}+\lambda_{s}^{2}\right)^{2}+\left(\frac{w}{\nu}\right)^{2}} \mathrm{e}^{-\left(\varsigma^{2}+\lambda_{s}{ }^{2}\right) t} \varsigma \sin (y \varsigma) d \varsigma, \\
& \tau_{s}(y, z, t)=\frac{2 \rho}{h} \sum_{s=0}^{\infty}(-1)^{s} \frac{\cos \left(\lambda_{s} z\right)}{\lambda_{s}} \mathrm{e}^{y D_{s}}\left[\sin \left(w t-y C_{s}\right)\right] \\
& -\frac{4 \rho}{\pi h} \sum_{s=0}^{\infty}(-1) \frac{\cos \left(\lambda_{s} z\right)}{\lambda_{s}} \\
& \int_{0}^{\infty} \frac{\frac{w}{\nu}}{\left(\varsigma^{2}+\lambda_{s}^{2}\right)^{2}+\left(\frac{w}{\nu}\right)^{2}} \mathrm{e}^{-\left(\varsigma^{2}+\lambda_{s}^{2}\right) t} \varsigma \sin (y \varsigma) d \varsigma .
\end{aligned}
$$




\section{Graphical Illustration and discussion}

In this discussion the unsteady Brinkman type fluid generated by the oscillation of the infinite bottom plate that applies an oscillating shear stress to the fluid which is viscous in nature is analyzed by using Fourier transformation, the solutions have been obtained and presented as sin and cos oscillations as well as for both the oscillations the solution are presented as steady and transient parts, which satisfies all the imposed and initial conditions. Fig.2 shows that for sin oscillation the velocity increases with the increasing of time while Fig.3 shows that for cos oscillation the velocity profile decreases with the increase of time parameter. Fig.4 exhibits that for sin oscillation the velocity increases with the increase in phase angle while Fig. 5 shows that for cos oscillation the velocity decreases with the increase in phase angle. Fig.6 and Fig. 7 are showing the behavior of sin and cos oscillations for different values of distances between

the walls (h) namely the velocities for the sin and cos oscillations are increasing by increasing the gaps between the walls. Fig. 8 and Fig. 9 are showing that the velocities for sin and cos oscillations are decreasing with the increasing values of Brinkman parameter $\beta$. Fig.10 and 11 show that if we increase the value of $\mathrm{h}$ to 1.6 the flow will behave like flowing over an infinite plate in sin oscillation, namely at this level the fluid in the center of the channel will the same on an infinite plate and the cos oscillation shows the same behavior as above at $h>0.25$. Fig. 12 shows that if we increase the time from 0.005 the graph of sin oscillation will overlap means after the said time the velocity profile for sin oscillation will become as steady motion of the fluid as well as in Fig.13 for cos oscillation shows the same behavior for time greater than 0.15 . 


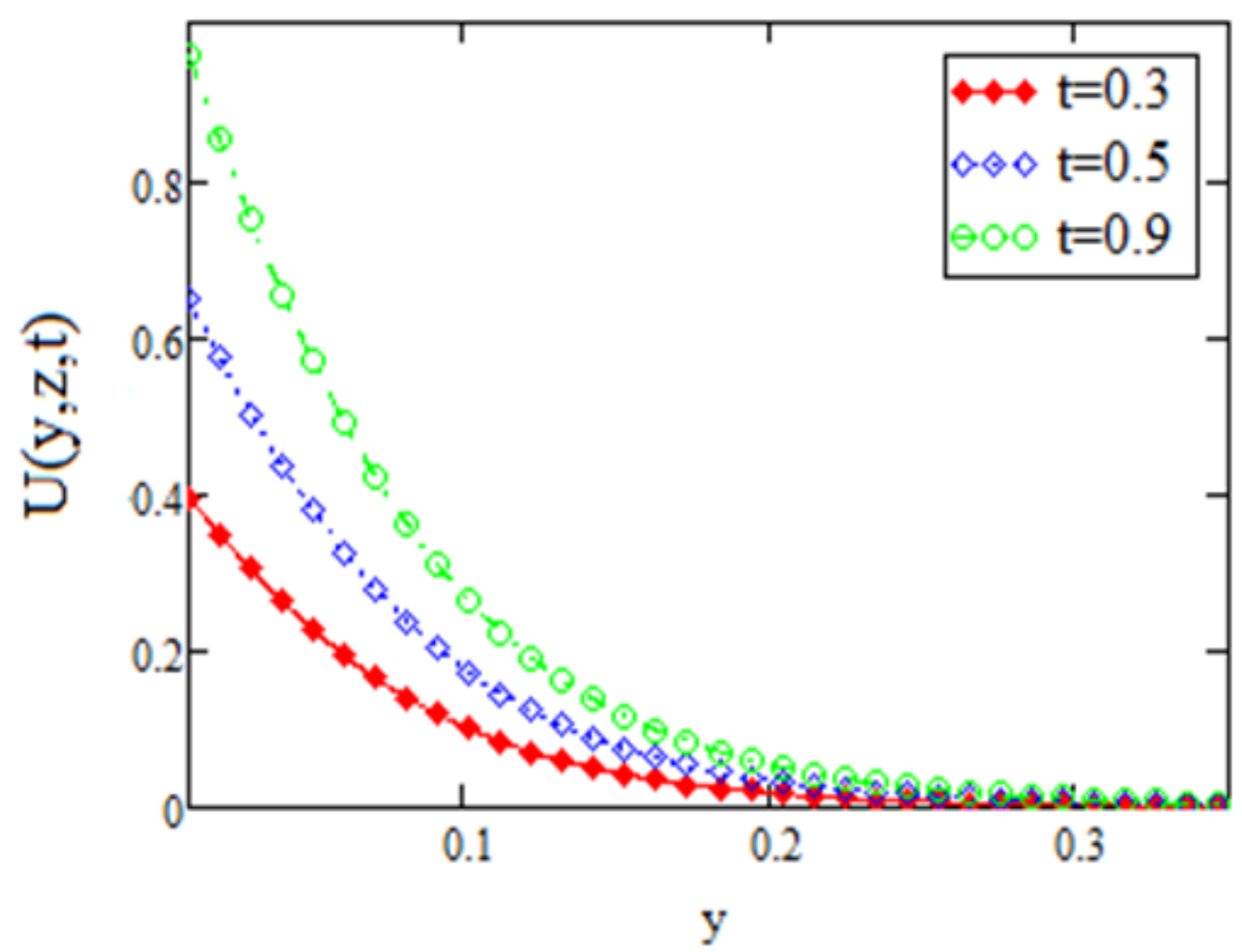

Figure 2: Velocities profiles $v_{s}(y, 0, t)$ shown by eq. (17). curves $v_{s}(y), v 1_{s}(y)$ and $v 2_{s}(y)$ with $w=1.5, h=$ $0.1, f=-0.8, \beta=0.7, \nu=0.1, \phi=0.7$ for the shear stress of the sin oscillation for different values of $\mathrm{t}$

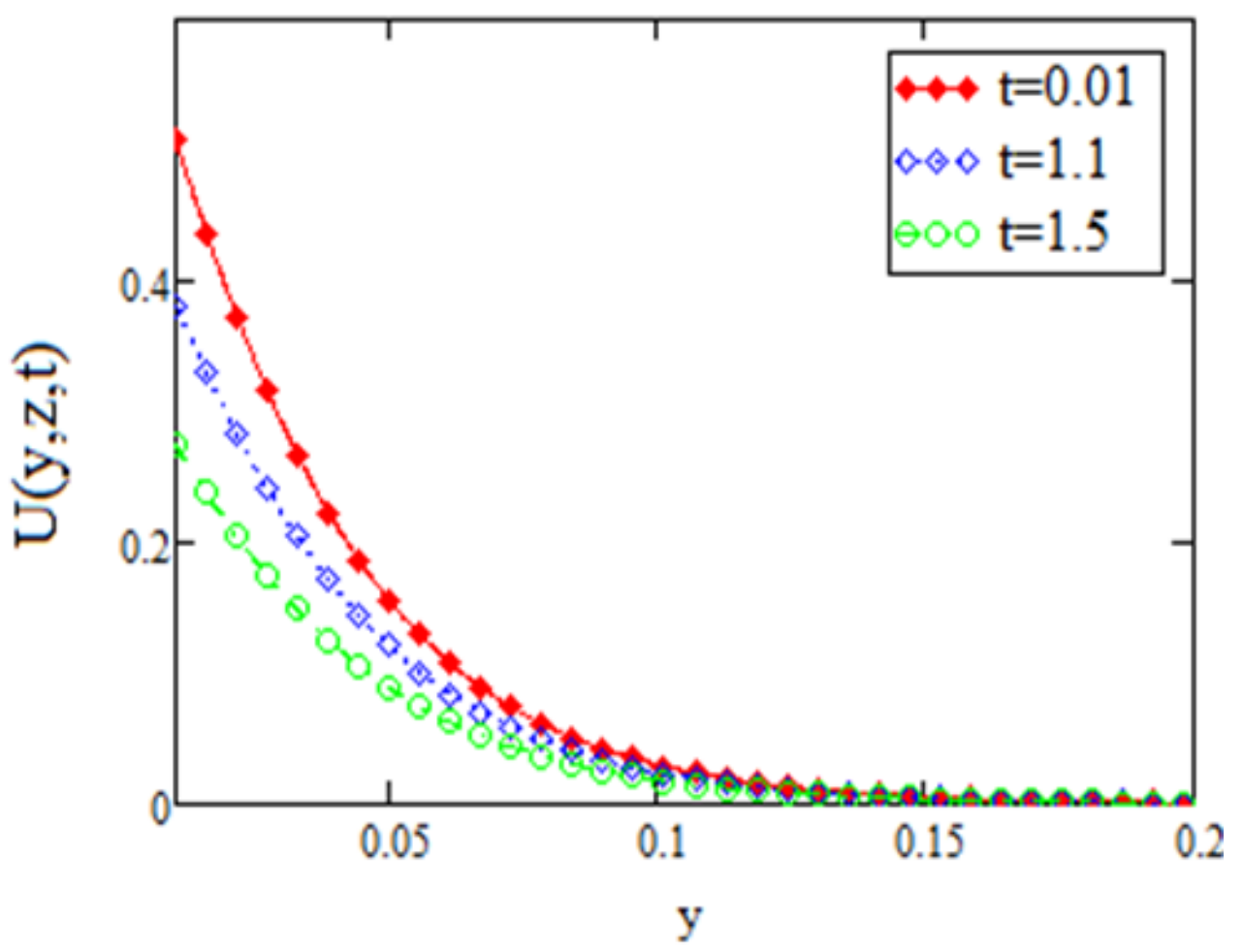

Figure 3: Velocities profiles $v_{c}(y, 0, t)$ shown by eq. (16). curves $v_{c}(y), v 1_{c}(y)$ and $v 2_{c}(y)$ with $w=0.7$, $h=$ 0.05, $f=-1, \phi=1.002, \beta=1, \nu=0.001$ for the shear stress of the cos oscillation for different values of $\mathrm{t}$ 


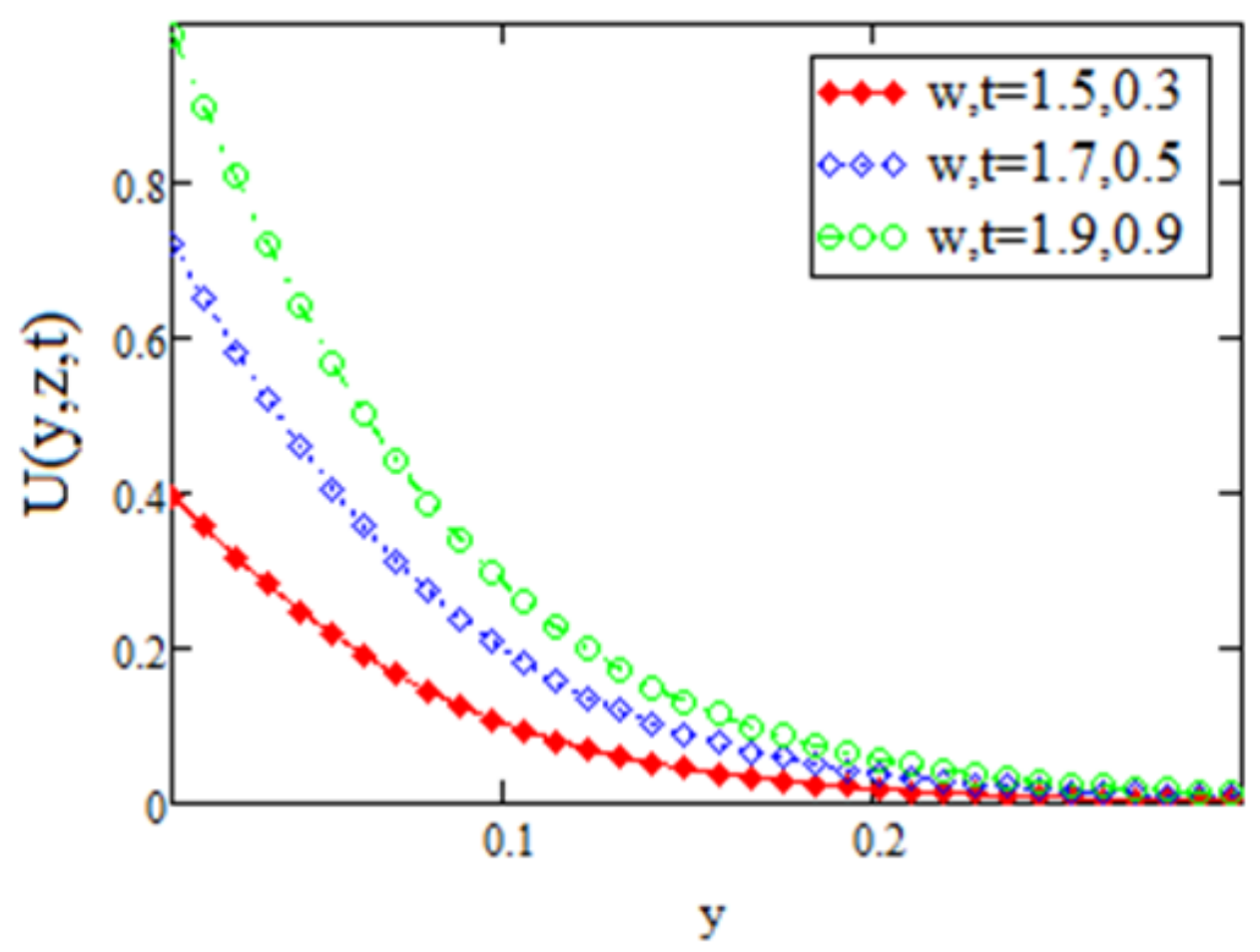

Figure 4: Velocities profiles $v_{s}(y, 0, t)$ shown by eq. (17). curves $v_{s}(y), v 1_{s}(y)$ and $v 2_{s}(y)$ with $\phi=0.7, h=$ $0.1, f=-0.8, \beta=0.7, \nu=0.01$ for the shear stress of the sin oscillation for different values of $\mathrm{w}$ and $\mathrm{t}$

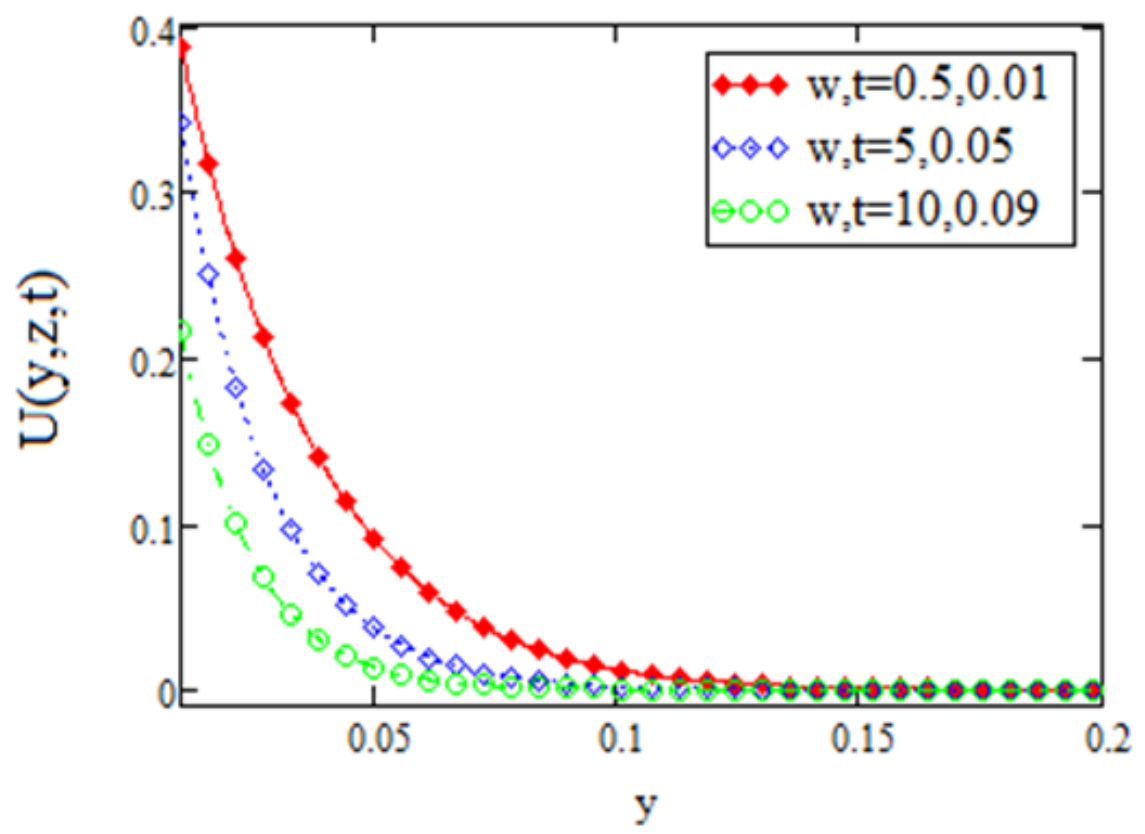

Figure 5: Velocities profiles $v_{c}(y, 0, t)$ shown by eq. (16). curves $v_{c}(y), v 1_{c}(y)$ and $v 2_{c}(y)$ with $\phi=1.5, h=$ $0.1, f=-1, \beta=1, \nu=0.001$ for the shear stress of the sin oscillation for different values of $\mathrm{w}$ and $\mathrm{t}$ 


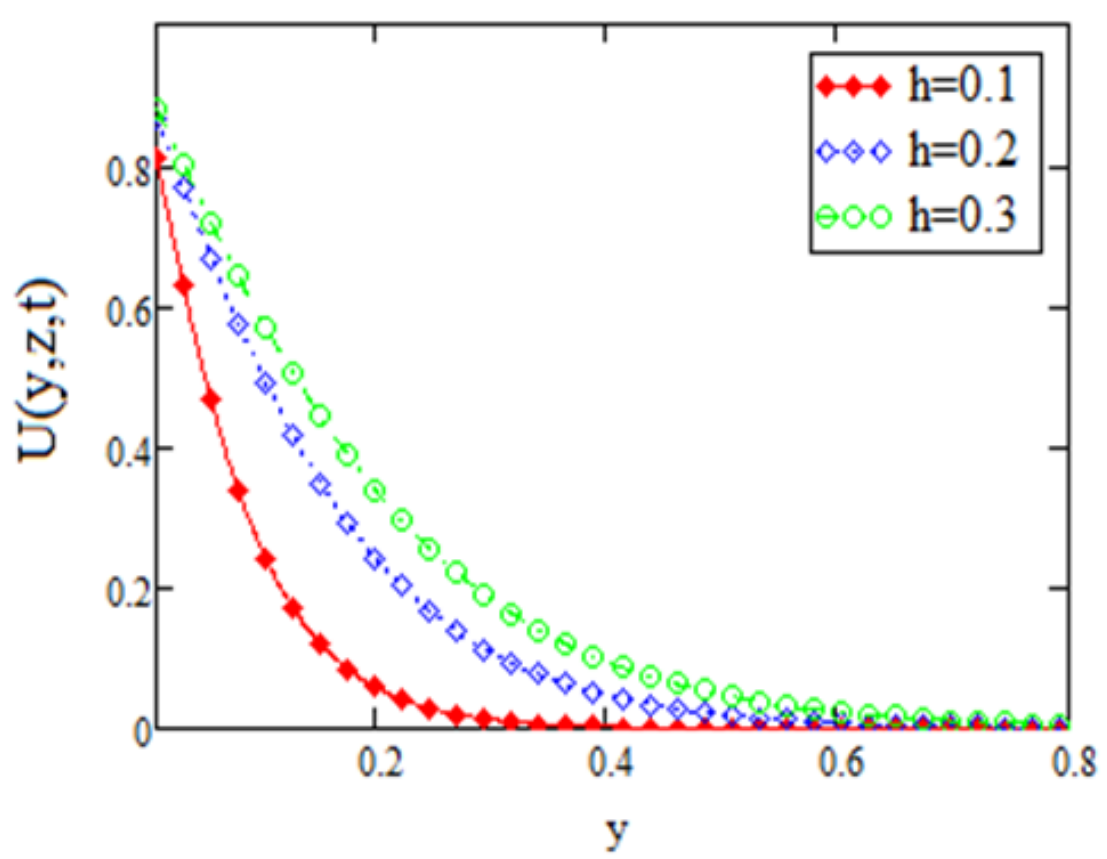

Figure 6: Velocities profiles $v_{s}(y, 0, t)$ shown by eq. (17). curves $v_{s}(y), v 1_{s}(y)$ and $v 2_{s}(y)$ with $\phi=0.7, f=$ $-1, \beta=0.7, \nu=0.01, t=0.5, w=5$ for the shear stress of the sin oscillation for different values of $h$

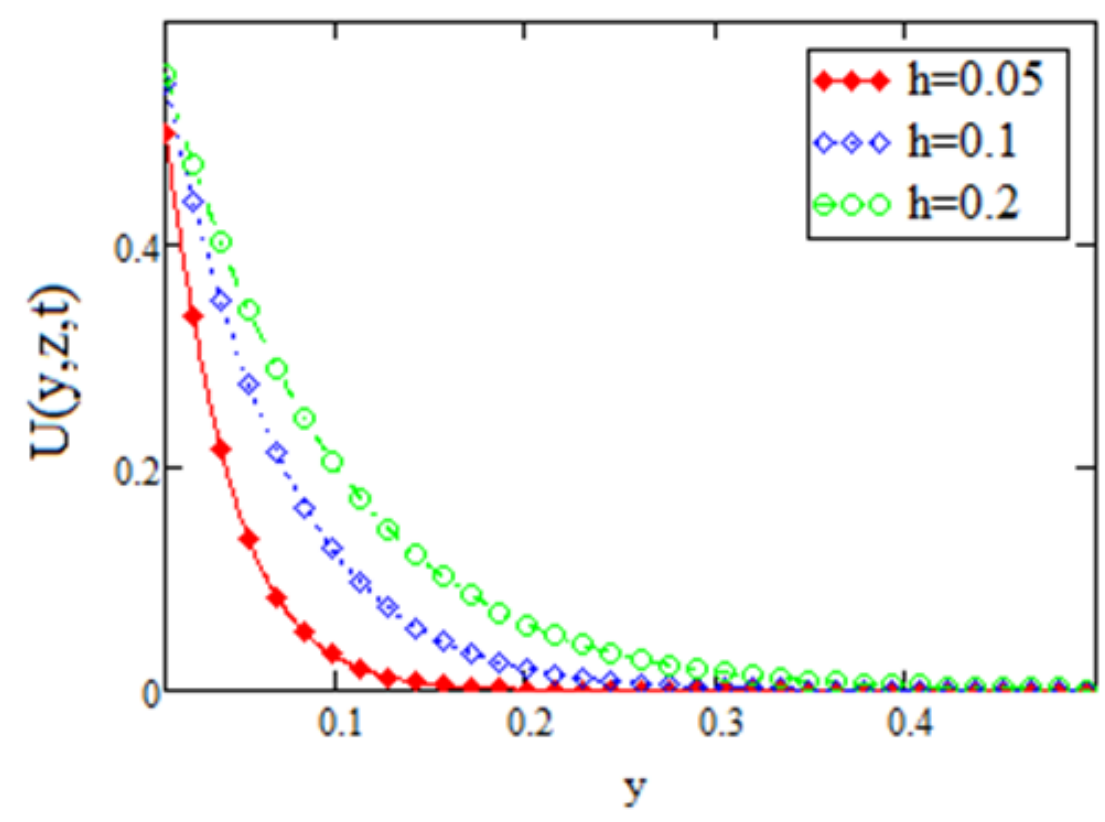

Figure 7: Velocities profiles $v_{c}(y, 0, t)$ shown by eq. (16). curves $v_{c}(y), v 1_{c}(y)$ and $v 2_{c}(y)$ with $w=0.5, t=$ $0.5, f=-1, \beta=1, \nu=0.01, \phi=1.5$ for the shear stress of the cos oscillation for different values of $h$ 


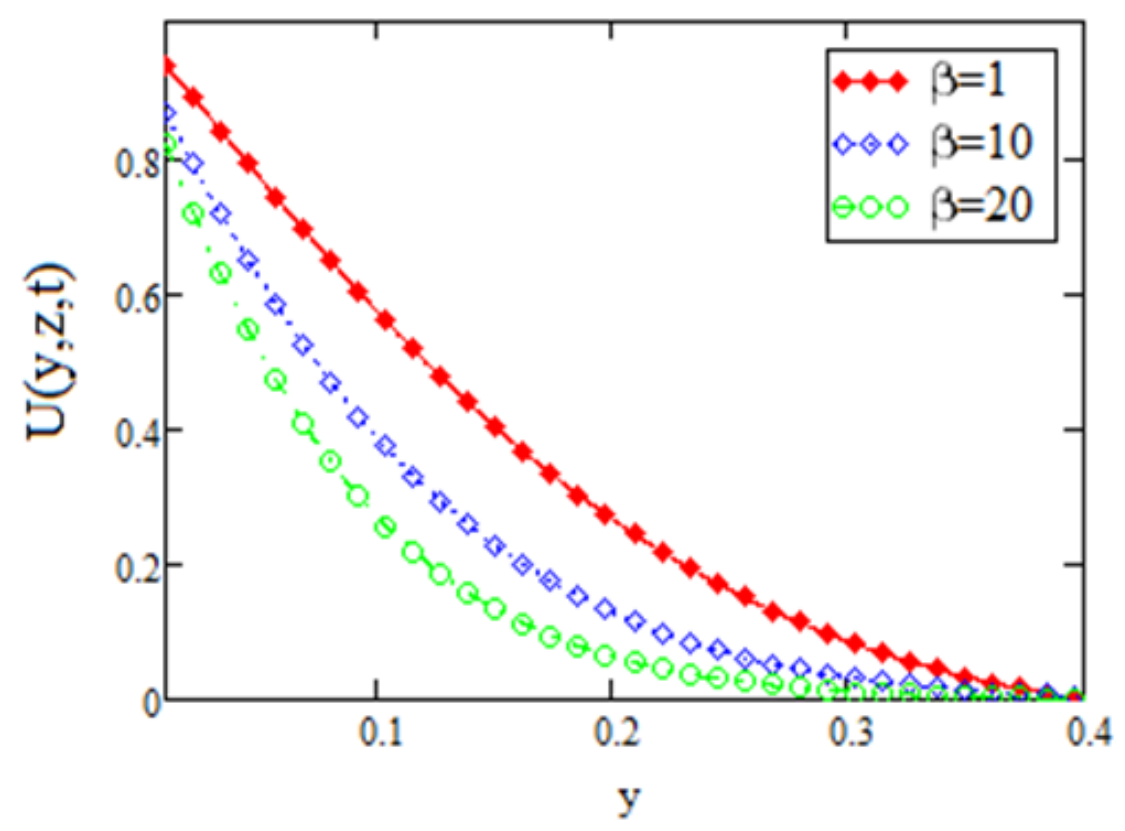

Figure 8: Velocities profiles $v_{s}(y, 0, t)$ shown by eq. (17). curves $v_{s}(y), v 1_{s}(y)$ and $v 2_{s}(y)$ with $w=5$, $h=$ $1, f=-1, t=0.5, \nu=0.1, \phi=0.7$ for the shear stress of the sin oscillation for different values of $\beta$

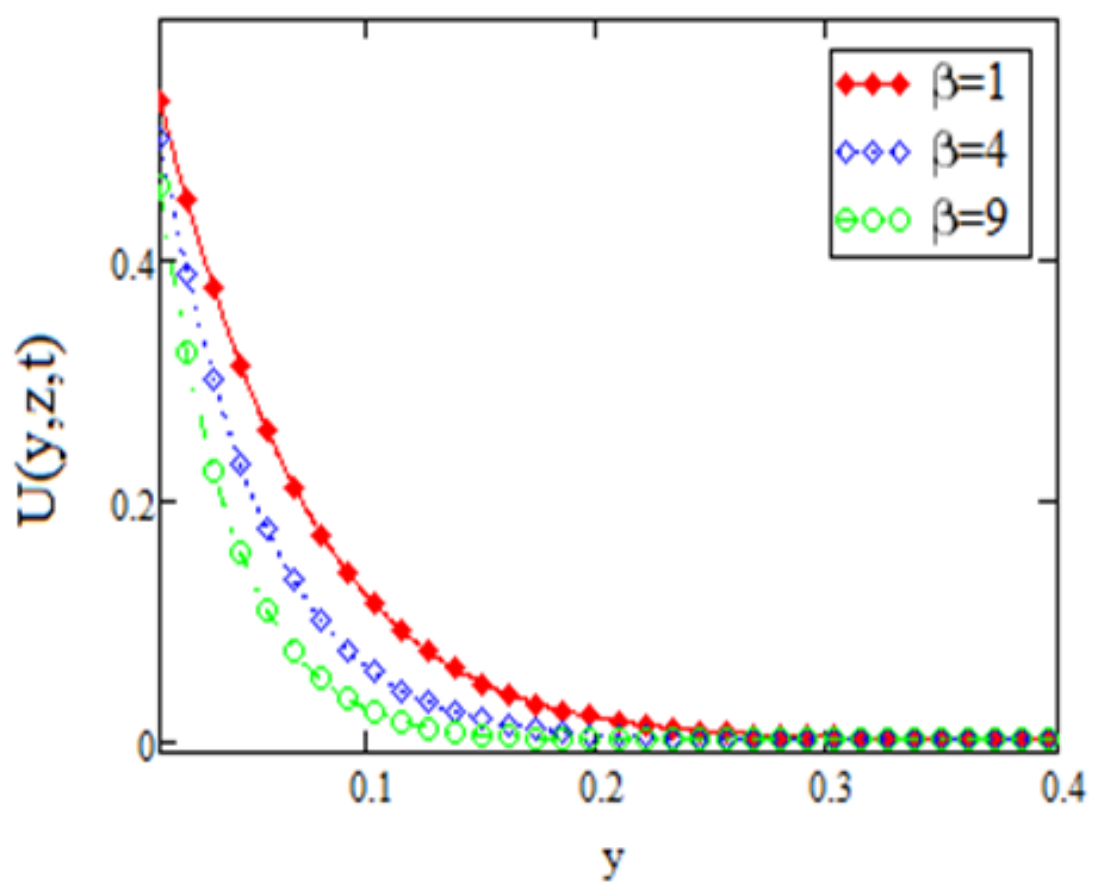

Figure 9: Velocities profiles $v_{c}(y, 0, t)$ shown by eq. (16). curves $v_{c}(y), v 1_{c}(y)$ and $v 2_{c}(y)$ with $w=0.5, h=$ $0.1, f=-5, t=0.5, \nu=0.01, \phi=1.5$ for the shear stress of the cos oscillation for different values of $\beta$ 


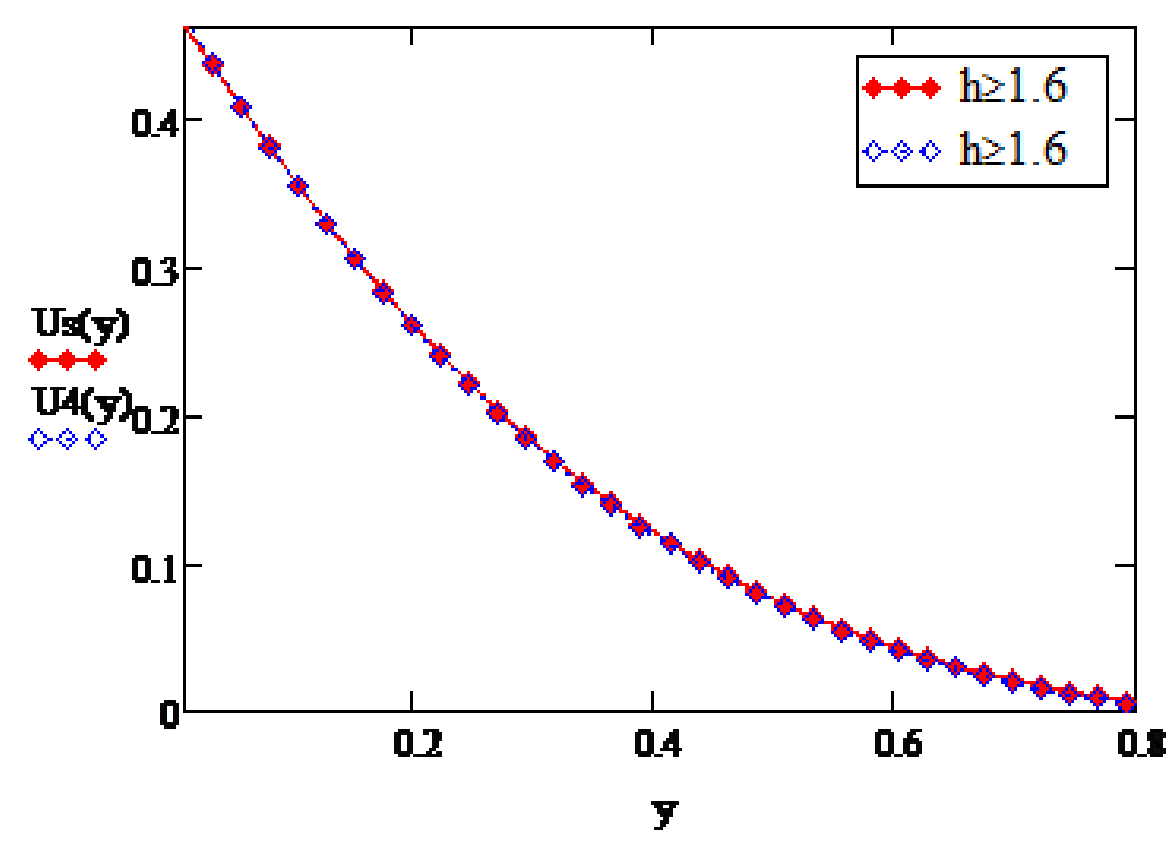

Figure 10: Velocities profiles $v_{s}(y, 0, t)$ shown by eq. (17). curves $v_{s}(y)$ and $v 2_{s}(y)$ with $w=0.01, \beta=1, f=$ $-5, t=5, \nu=0.001002$ for the shear stress of the sin oscillation the curves will overlap for value of $h \geq 1.6$

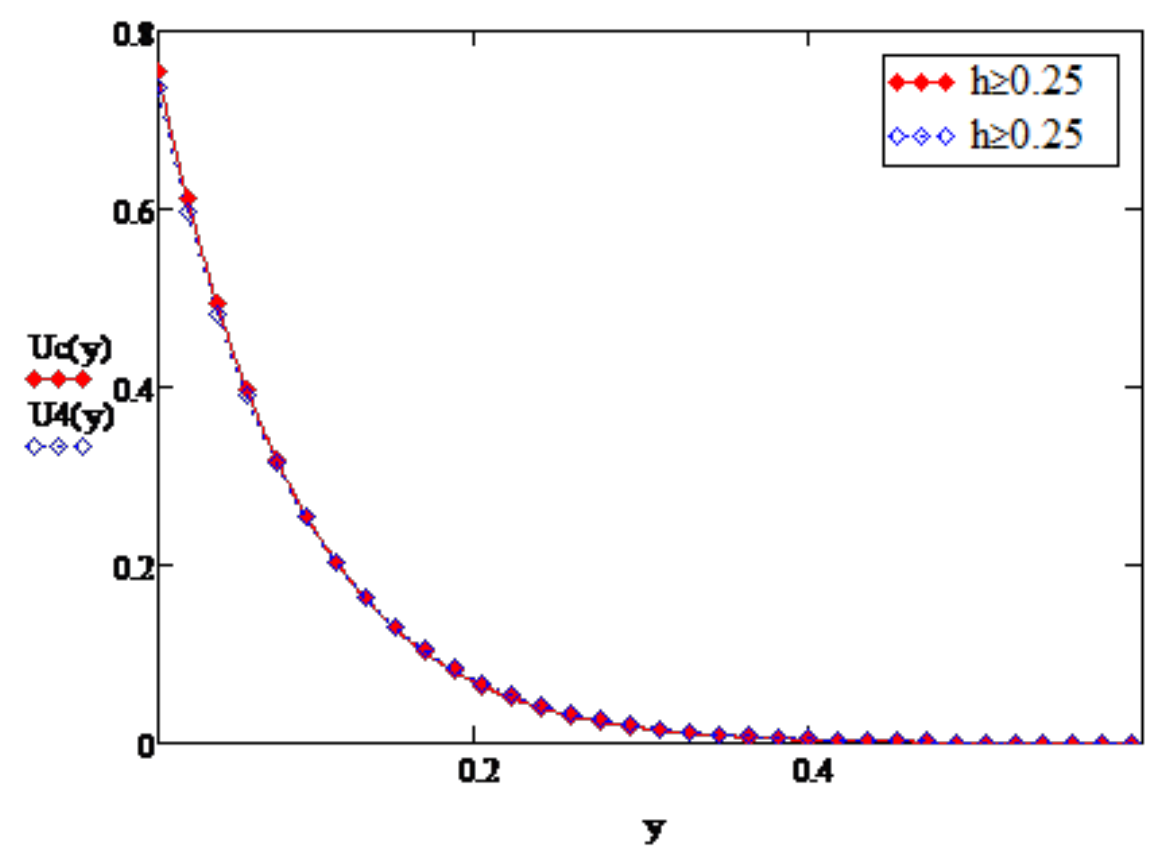

Figure 11: Velocities profiles $v_{c}(y, 0, t)$ shown by eq. (16). curves $v_{c}(y)$ and $v 2_{c}(y)$ with $w=0.01, \beta=1, f=$ $-5, t=1, \nu=0.001002$ for the shear stress of the sin oscillation the curves will overlap for value of $h \geq 0.25$ 


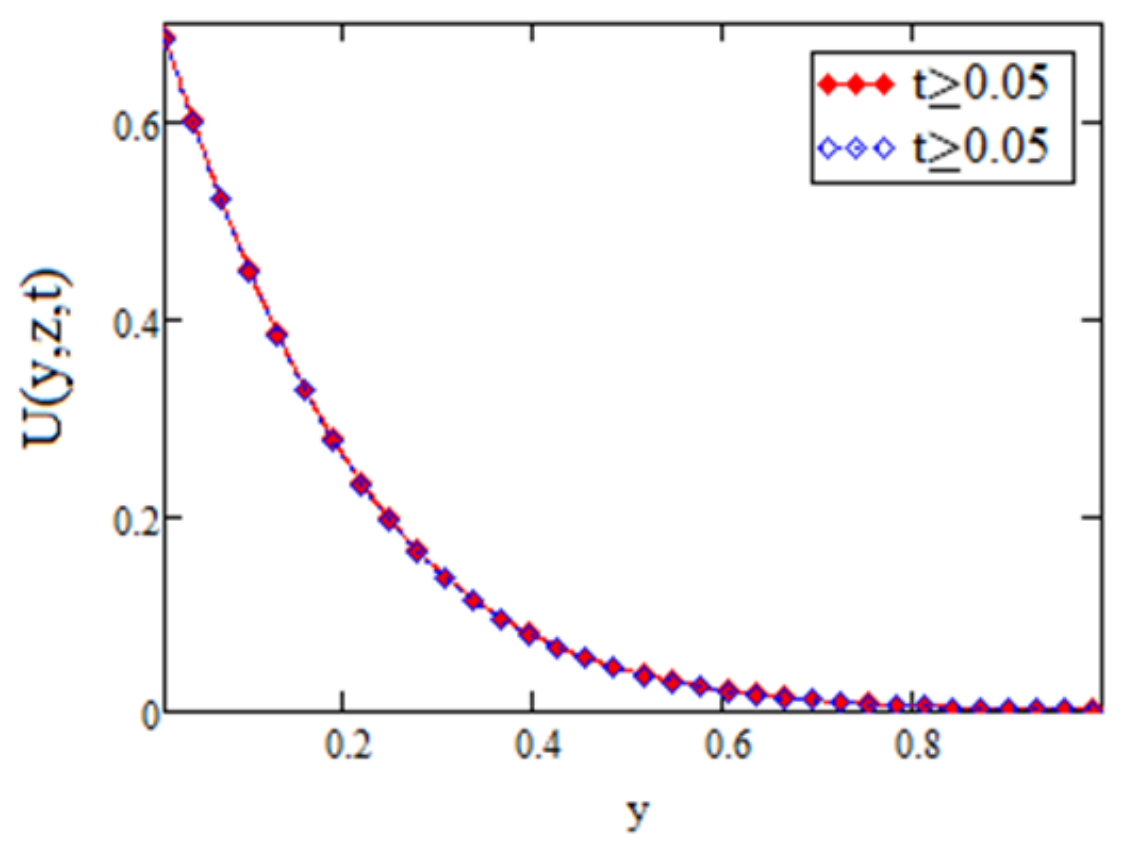

Figure 12: The time required when the velocities profiles $v_{s}(y, 0, t)$ shown by eq. (17). curves $v_{s}(y)$ and $v 3_{s}(y)$ with $w=5, h=0.3 \beta=15, f=-5, t \geq 0.05, \nu=3.5, \phi=1.5$ for the shear stress of the sin oscillation the curves will overlap for value of $t \geq 0.05$

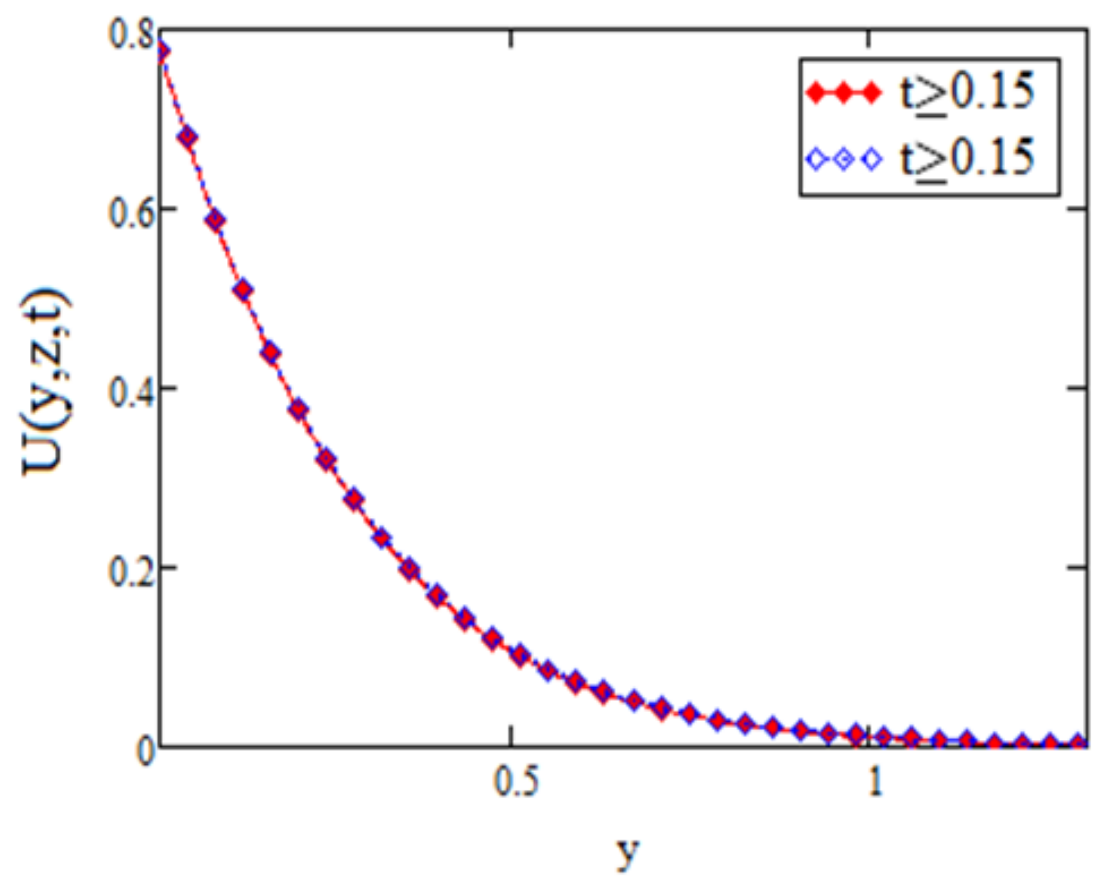

Figure 13: The time required when the velocities profiles $v_{c}(y, 0, t)$ shown by eq. (16). curves $v_{c}(y)$ and $v 3_{c}(y)$ with $w=0.01, h=0.5 \beta=0.1, f=-1, t \geq 0.15, \nu=0.01, \phi=1.2$ for the shear stress of the cos oscillation the curves will overlap for value of $t \geq 0.15$ 


\section{Conclusion}

Unsteady Brinkman type of flow over an infinite plate between side walls is being investigated while the flow is produced by oscillating of the infinite plate. The exact solution is being obtained by using Fourier transformation in terms of sin and cos oscillation and presented as steady and transient part. The following conclusions are extracted

(i) Change in velocity occurs due to change in time, angle, distance between side walls. in sin oscillation the velocity increases with increase in time, angle or distance between side walls(h) while in cos oscillation the velocity decreases with increase in above parameters.

(ii) By decreasing the value of Brinkman parameter namely $\beta \rightarrow o$ our results tends to the exact solution obtained by Fetecua [16]

(iii) after a specific time the flow becomes steady both in sin and cos oscillation. (iv) after a particular distance between side walls the flow becomes unaffected of walls.

\section{References}

[1] H. Brinkman, A calculation of the viscous force exerted by a flowing fluid on a dense swarm of particles, Applied Scientific Research 1 (1) (1949) $27-34$.

[2] H. Brinkman, On the permeability of media consisting of closely packed porous particles, Applied Scientific Research 1 (1) (1949) 81-86.

[3] F. Ali, M. Gohar, I. Khan, Mhd flow of water-based brinkman type nanofluid over a vertical plate embedded in a porous medium with variable surface velocity, temperature and concentration, Journal of Molecular Liquids 223 (2016) 412-419.

[4] S. V. Varma, M. Babu, A brinkman model for mhd viscous incompressible flow through a porous channel, Indian Journal of Pure and Applied Mathematics 16 (7) (1985) 796-806.

[5] C. Hsu, P. Cheng, The brinkman model for natural convection about a semi-infinite vertical flat plate in a porous medium, International Journal of Heat and Mass Transfer 28 (3) (1985) 683-697. 
[6] H. Darcy, Les fontaines publiques de la ville de Dijon: exposition et application..., Victor Dalmont, 1856.

[7] K. Rajagopal, On a hierarchy of approximate models for flows of incompressible fluids through porous solids, Mathematical Models and Methods in Applied Sciences 17 (02) (2007) 215-252.

[8] F. Ali, I. Khan, S. Shafie, et al., A note on new exact solutions for some unsteady flows of brinkman-type fluids over a plane wall, Zeitschrift für Naturforschung A 67 (6-7) (2012) 377-380.

[9] R. Araya, C. Harder, A. H. Poza, F. Valentin, Multiscale hybrid-mixed method for the stokes and brinkman equations-the method, Computer Methods in Applied Mechanics and Engineering.

[10] L. Tao, On combined free and forced convection in channels, ASME J. Heat Transfer 82 (3) (1960) 233-238.

[11] J. Hartmann, Hydrodynamic i. theory of the laminar flow of an electrically conducting liquid in a homogeneous magnetic field, Kgl Danske Videns Kabernes Selskab Math. Fys. Med 15 (6).

[12] J. C. Umavathi, J. P. Kumar, M. A. Sheremet, Heat and mass transfer in a vertical double passage channel filled with electrically conducting fluid, Physica A: Statistical Mechanics and its Applications 465 (2017) 195-216.

[13] W. Akhtar, C. Fetecau, V. Tigoiu, C. Fetecau, Flow of a maxwell fluid between two side walls induced by a constantly accelerating plate, Zeitschrift für angewandte Mathematik und Physik 60 (3) (2009) 498-510.

[14] D. Vieru, C. Fetecau, A. Sohail, Flow due to a plate that applies an accelerated shear to a second grade fluid between two parallel walls perpendicular to the plate, Zeitschrift für Angewandte Mathematik und Physik (ZAMP) 62 (1) (2011) 161-172.

[15] Y. Yao, Y. Liu, Some unsteady flows of a second grade fluid over a plane wall, Nonlinear Analysis: Real World Applications 11 (5) (2010) 4442-4450.

[16] C. Fetecau, D. Vieru, C. Fetecau, Effect of side walls on the motion of a viscous fluid induced by an infinite plate that applies an oscillating shear stress to the fluid, Open Physics 9 (3) (2011) 816-824. 
[17] C. Fetecau, C. Fetecau, M. Rana, General solutions for the unsteady flow of second-grade fluids over an infinite plate that applies arbitrary shear to the fluid, Zeitschrift für Naturforschung A 66 (12) (2011) 753-759.

[18] D. Vieru, C. Fetecau, M. Rana, Starting solutions for the flow of second grade fluids in a rectangular channel due to an oscillating shear stress, in: AIP Conference Proceedings, Vol. 1450, AIP, 2012, pp. 45-54.

[19] M. Rana, N. Shahid, A. A. Zafar, Effects of side walls on the motion induced by an infinite plate that applies shear stresses to an oldroyd-b fluid, Zeitschrift für Naturforschung A 68 (12) (2013) 725-734.

[20] A. Sohail, D. Vieru, M. Imran, Influence of side walls on the oscillating motion of a maxwell fluid over an infinite plate, Mechanics 19 (3) (2013) 269-276.

[21] S. U. Haq, A. ur Rahman, I. Khan, F. Ali, S. I. A. Shah, The impact of side walls on the mhd flow of a second-grade fluid through a porous medium, Neural Computing and Applications (2016) 1-7. 